\title{
Charge Carrier Dynamics and Broad Wavelength Tunable Amplified Spontaneous Emission in $\mathrm{Zn}_{\mathrm{x}} \mathrm{Cd}_{1-x} \mathrm{Se}$ Nanowires
}

Xiaojun Li, ${ }^{\star}$ Qi Wei, ${ }^{\star}$ Kaiyang Wang, Shaomin Peng, Tanghao Liu, Guichuan Xing, ${ }^{*}$ and Zikang Tang*

Institute of Applied Physics and Materials Engineering, University of Macau, Avenida da Universidade, Taipa, Macau 999078, PR China

E-mails of Corresponding Authors: gcxing@um.edu.mo, zktang@um.edu.mo

\section{Experiments}

Preparation of samples: All the $\mathrm{Zn}_{x} \mathrm{Cd}_{1-x} \mathrm{Se}(0 \leq x \leq 1)$ NWs were grown on Si substrates by vapor-liquid-solid growth method in a horizontal tube furnace using starting materials of $\mathrm{ZnSe}$ powder (99.99\%), CdSe powder (99.99\%). The ZnSe and CdSe powders contained in two quartz boats respectively that were placed in two side regions of the quartz tube. And the silicon substrate coated with a layer of $\mathrm{Au}(5 \mathrm{~nm})$ was then placed at the center that is about $10 \mathrm{~cm}$ far away from above two sources. The Au film served as catalytic sites for the nucleation and growth of the NWs. The tube furnace was heated to $700{ }^{\circ} \mathrm{C}$ (with a heating rate of $5{ }^{\circ} \mathrm{C} \mathrm{min}^{-1}$ ) for $30 \mathrm{~min}$ and then down to room temperature spontaneously in the furnace with the power switched off. In order to prevent the samples from being oxidized, all heating and cooling processes were conducted in the Ar gas flowing at a rate of $50 \mathrm{sccm}$. The sponge-like products with color ranging from canary yellow to dark brown were observed on the silicon wafer.

Characterizations: The morphologies of obtained samples were observed by scanning electron microscope (SEM) (JEOL JSM-7001F, operating at $10 \mathrm{KV}$ ). The crystallization quality of these nanostructures was detected and analyzed by transmission electron microscopy (TEM) (JEOL JEM-2100). The PL and lifetime of $\mathrm{Zn}_{x} \mathrm{Cd}_{1-x} \mathrm{Se}(0 \leq x \leq 1) \mathrm{NWs}$ were collected under $400 \mathrm{~nm}$ laser pulses in a typical backscattering geometry by a pair of lenses, which was split by a beamsplitter and directed to a spectrometer (Acton, Spectra Pro 2300i) equipped with a charge couple device camera (Princeton Instruments, Pixis 400B) and an Optronis Optoscope streak camera system for time resolved photoluminescence (TRPL) measurements. The excitation power dependent one photon $(400 \mathrm{~nm})$ and two photon $(800 \mathrm{~nm})$ pumped emission spectra are 
collected from multiple $\mathrm{Zn}_{x} \mathrm{Cd}_{1-x} \mathrm{Se}(0 \leq x \leq 1) \mathrm{NWs}$ in a macroscopical region (light spot diameter is about $50 \mu \mathrm{m}$ ). The excitation pulses and emission light are coupled and collected through slit of spectrometer, and spectrally resolved by the spectrometer and charge coupled device (CCD) detector. $800 \mathrm{~nm}$ laser pulses come from the regenerative amplifier and $400 \mathrm{~nm}$ laser pulses were obtained with a beta barium borate (BBO) crystal. All the above measurements were performed at room temperature. In addition, temperature dependent optical studies were performed in an open-cycle helium cryostat, with the output pulses from a coherent legend regenerative amplifier (400 nm, $150 \mathrm{fs}$, and $1 \mathrm{kHz}$ laser pulses) as the excitation source.

\section{Supporting Figures and Discussions}
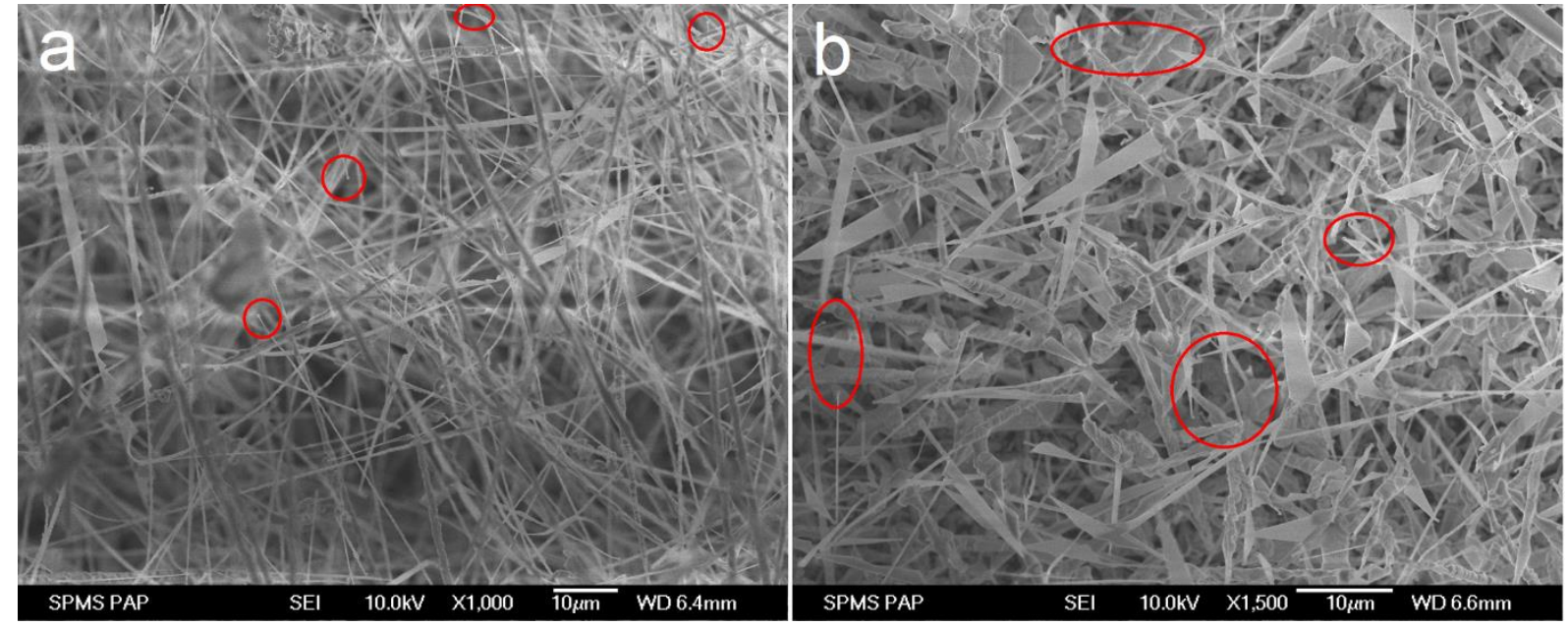

Figure S1. (a) SEM image of $\mathrm{Zn}_{0.5} \mathrm{Cd}_{0.5} \mathrm{Se} \mathrm{NWs}$ with a small globule at the tip. (b) SEM image of $\mathrm{Zn}_{0.2} \mathrm{Cd}_{0.8} \mathrm{Se} \mathrm{NWs} /$ nanoplates with a small globule at the tip.
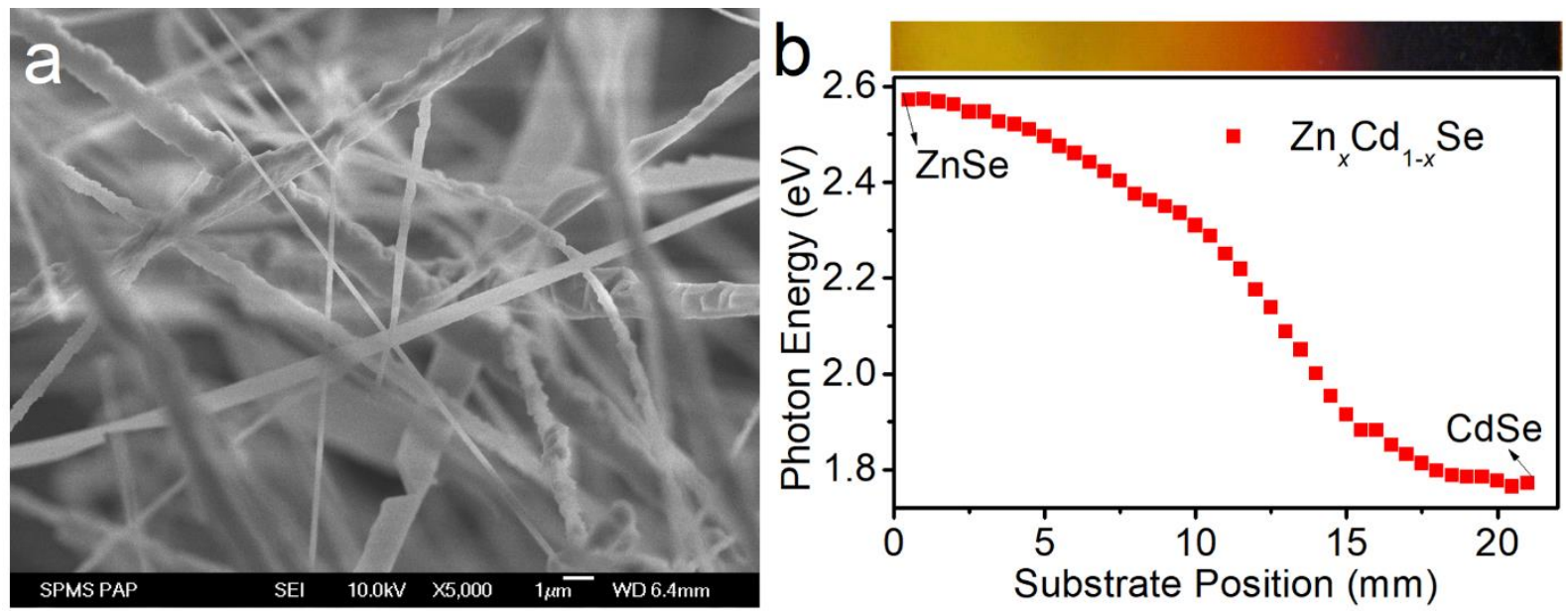

Figure S2. (a) Local amplified SEM image of $\mathrm{Zn}_{0.5} \mathrm{Cd}_{0.5} \mathrm{Se}$ NWs. (b) PL peak energy as a function of substrate length coordinates. The corresponding optical images of these samples are shown above where there is a color variation from canary yellow $(\mathrm{ZnSe})$ to dark brown $(\mathrm{CdSe})$ along the substrate length. 

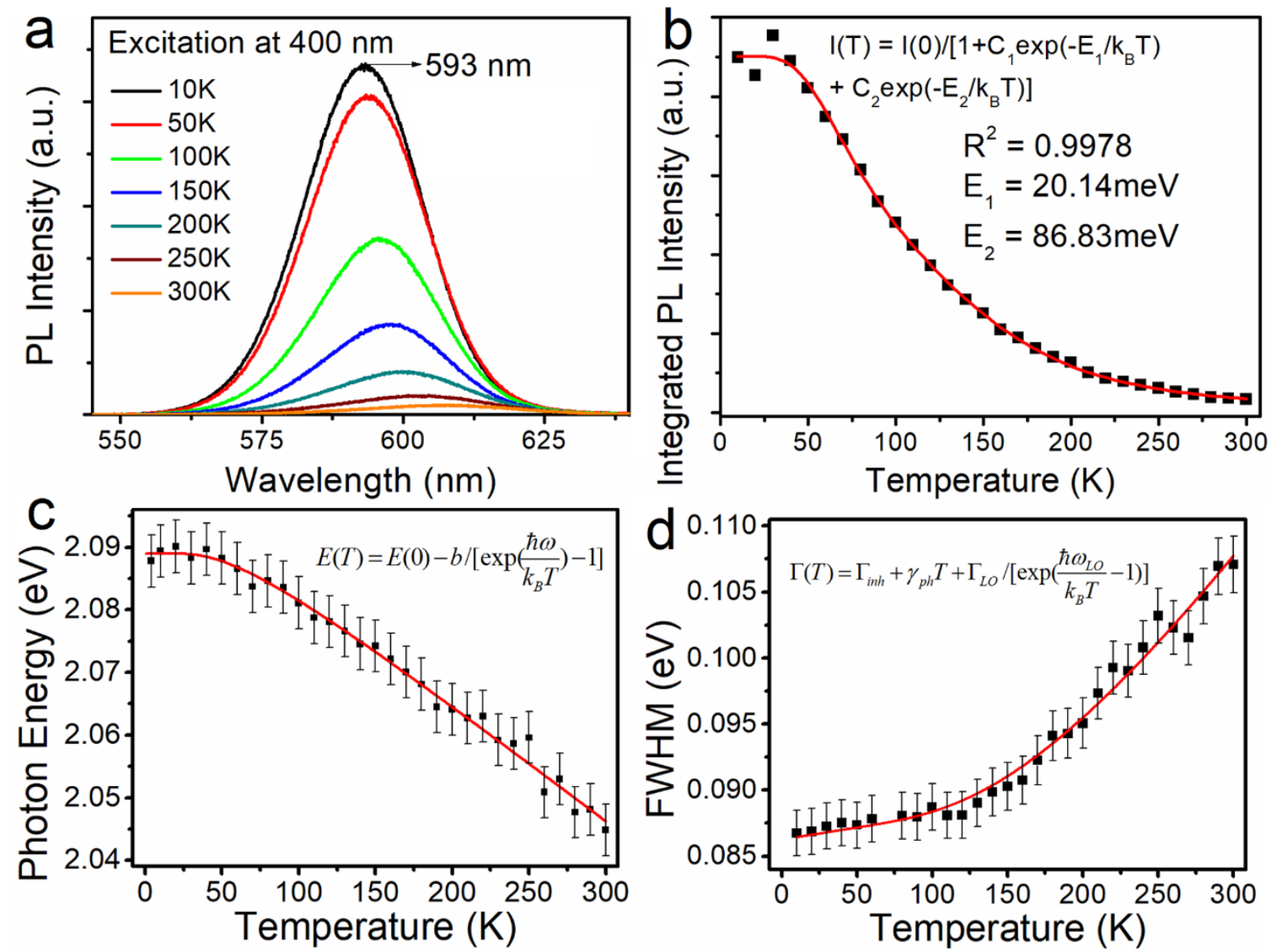

Figure S3. (a) The evolution of PL spectra of $\mathrm{Zn}_{0.5} \mathrm{Cd}_{0.5} \mathrm{Se}$ with the increase of temperature; (b) Integrated PL intensity with the increase of temperature; (c) Integrated photon energy with the increase of temperature; (d) Integrated fwhm with the increase of temperature.

Figure S3a shows temperature-dependent PL spectra of $\mathrm{Zn}_{0.5} \mathrm{Cd}_{0.5} \mathrm{Se}(x=0.5)$ NWs with increasing temperature from $10 \mathrm{~K}$ to $300 \mathrm{~K}$. Both the PL intensity and photon energy decrease with the increase of temperature. The integrated PL intensity for two thermal quenching processes can be fitted by Arrhenius equation, as follow: ${ }^{1}$

$I(T)=I(0) /\left[1+c_{1} \exp \left(-\frac{E_{1}}{k_{B} T}\right)+c_{2} \exp \left(-\frac{E_{2}}{k_{B} T}\right)\right]$

Here $I(0)$ is the initial intensity, $I(T)$ is the intensity at a given temperature $T, c_{1}$ and $c_{2}$ are the relative ratios of the two non-radiative recombination processes, $E_{1}$ and $E_{1}$ are the different activation energies for the two different thermal quenching processes, and $k_{B}$ is Boltzmann's constant. According to equation (1), the $I(T)$ deceases with the increase of $T$, as shown in Figure $\mathrm{S} 3 \mathrm{~b}$. The fitting result indicates that the activation energies of $E_{1}=20.14 \mathrm{meV}$ and $E_{2}=86.83$ $\mathrm{meV}$. With the increase of temperature, bound exciton complexes will be split into free charges because the crystal lattice vibrational energy becomes effective and dominates over the exciton binding energy. Hence, $E_{1}$ is the activation energy required (exciton binding energy) for 
dissociation of free excitons, which is correspond to free exciton binding energy of ZnSe (19 $\mathrm{meV})$ and $\mathrm{CdSe}(15 \mathrm{meV}){ }^{2,3}$ With the further increase of temperature, PL intensity decreases more rapidly than the decrease caused by thermal-activated free exciton dissociation. Therefore, $E_{2}$ is the activation energy required for non-radiative charge carrier trapping losses stem from thermal-assisted capture of carriers at some defect states.

Figure S3c indicates the bandgap (photon energy) decreases with the increase of temperature, which is caused by the thermal expansion of the crystal lattice and exciton-phonon interactions. The relationship of temperature dependent photon energy $E(T)$ can be expressed by BoseEinstein equation, ${ }^{4}$

$$
E(T)=E(0)-b /\left[\exp \left(\frac{\hbar \omega}{k_{B} T}\right)-1\right]
$$

Here $E(T)$ is the photon energy at a given temperature $T, E(0)$ is the initial photon energy, $b$ is proportionality coefficient, $\hbar \omega$ is the effective phonon energy, and $k_{B}$ is Boltzmann's constant. According to equation (2), the $E(T)$ decreases with the increase of $T$. As shown in Figure S3c, the room temperature $E(300 \mathrm{~K})$ is $2.046 \mathrm{eV}$ and $E(0 \mathrm{~K})=2.089 \mathrm{eV}$. The fitting result indicates that the effective phonon energy is $14.05 \mathrm{meV}$. After accounting for the exciton binding energy of $20.14 \mathrm{meV}$, which get the accurate bandgaps of $\mathrm{Zn}_{0.5} \mathrm{Cd}_{0.5} \mathrm{Se}$ are $2.109 \mathrm{eV}$ at $0 \mathrm{~K}$ and 2.066 $\mathrm{eV}$ at $300 \mathrm{~K}$.

The carrier and phonons coupling are closely related to the full width at half maximum (fwhm) of the materials. As shown in Figure S3d, the fwhm of $\mathrm{Zn}_{0.5} \mathrm{Cd}_{0.5} \mathrm{Se} \mathrm{NW}$ s increases with the increase of the temperature from $4 \mathrm{~K}$ to $300 \mathrm{~K}$. The broadening of the emission band origin from inhomogeneous (crystal imperfections) and homogeneous effects (longitudinal optical (LO) phonon and longitudinal acoustic (LA) phonon only intraband scattering). In the absence of the hot electron effects, the temperature dependent fwhm of the emission spectra can be expressed by following equation, ${ }^{4}$

$$
\Gamma(T)=\Gamma_{i n h}+\gamma_{p h} T+\Gamma_{L O} /\left[\exp \left(\frac{\hbar \omega_{L O}}{k_{B} T}-1\right)\right]
$$

where $\Gamma_{\text {inh }}$ is the inhomogeneous broadening factor, $\gamma_{\mathrm{ph}}$ is carrier-LA phonon coupling strength, $\Gamma_{\mathrm{LO}}$ is carrier-LO phonon coupling strength, and $\hbar \omega_{\mathrm{LO}}$ is the LO phonon energy. At very low temperature, the dominant contribution of the fwhm stems from inhomogeneous broadening because the phonons are not active. According to the fitting results based on ignoring the hot electron effects, it can be obtained that $\Gamma_{\mathrm{inh}}=86.26 \mathrm{meV}, \gamma_{\mathrm{ph}}=18 \mu \mathrm{eV} \mathrm{K}{ }^{-1}, \hbar \omega_{\mathrm{LO}}=48.9 \mathrm{meV}$ and $\Gamma_{\mathrm{LO}}=90.87 \mathrm{meV}$. 
Table S1. Calculated lattice parameter, band gap, transition energy, dipole oscillator strength and radiative lifetime for $\mathrm{Zn}_{x} \mathrm{Cd}_{1-x} \mathrm{Se}$ by GGA-PBE. The lifetimes are calculated with the index of refraction $(n=2.5)$. (Experimental data are in the brackets)

\begin{tabular}{llllllll}
\hline \multirow{2}{*}{ Semiconductors } & \multicolumn{2}{l}{ Lattice Parameter $(\AA)$} & Band & Gap & \multicolumn{2}{l}{ Transition } & \multicolumn{2}{l}{ Lifetime } \\
\cline { 2 - 3 } \cline { 5 - 6 } & & $\mathrm{c}$ & $(\mathrm{eV})$ & $\mathrm{c}$ & $\mathrm{E}$ & $\mathrm{f}$ & $(\mathrm{ps})$ \\
\hline $\mathrm{CdSe}$ & $4.38(4.30)$ & $7.16(7.01)$ & $1.44(1.77)$ & 1.52 & 0.0019 & 3718 \\
$\mathrm{Cd}_{0.75} \mathrm{Zn}_{0.25} \mathrm{Se}$ & 4.30 & 7.07 & 1.47 & 1.78 & 0.0063 & 803 \\
$\mathrm{Cd}_{0.5} \mathrm{Zn}_{0.5} \mathrm{Se}$ & 4.21 & 6.95 & 1.55 & 2.13 & 0.0094 & 363 \\
$\mathrm{Cd}_{0.25} \mathrm{Zn}_{0.75} \mathrm{Se}$ & 4.12 & 6.81 & 1.70 & 2.14 & 0.0154 & 214 \\
$\mathrm{ZnSe}$ & $4.05(3.82)$ & $6.65(6.63)$ & $2.18(2.58)$ & 2.25 & 0.0562 & 51 \\
\hline
\end{tabular}

The electronic and optical properties of $\mathrm{Zn}_{x} \mathrm{Cd}_{1-x} \mathrm{Se}(x=0,0.25,0.5,0.75,1)$ were investigated by density functional theory (DFT) with generalized gradient approximation (GGA) based on Perdew-Burker-Ernzerhof (PBE) functional calculations using the QuantumESPRESSO software package. ${ }^{5,6}$ The Vanderbilt ultrasoft pseudopotentials were used to describe the electron-ion interactions for $\mathrm{Cd}(4 s, 5 d, 5 p), \mathrm{Zn}(3 s, 3 p, 3 d, 4 s, 4 p)$ and $\mathrm{Se}(4 s, 4 p)$ atoms. The electronic wavefunctions and charge density were expanded with an energy cutoff of $40 \mathrm{Ry}$ and $280 \mathrm{Ry}$, respectively. For calculations of bulk $\mathrm{Zn}_{x} \mathrm{Cd}_{1-x} \mathrm{Se}$, the integration over the Brillouin Zone was performed using a k-points grid of $8 \times 8 \times 8$ during the cell optimization and electronic properties calculations. All bulk geometries were optimized with the method of Broyden-Fletcher-Goldfarb-Shanno (BFGS) until all the forces on all atoms became lower than $0.02 \mathrm{eV} / \AA$ and total energy difference between two optimization steps of the minimization procedures was less than $10^{-4} \mathrm{eV}$. The calculated lattice parameter, band gap, transition energy, dipole oscillator strength and radiative lifetime for $\mathrm{Zn}_{x} \mathrm{Cd}_{1-x} \mathrm{Se}(x=0,0.25,0.5,0.75,1)$ are summarized in Table 1. We have obtained a lattice constant of $\mathrm{a}=4.38 \AA$ and $\mathrm{c}=7.16 \AA$ for $\mathrm{CdSe}$, which is close to the experimental values of $\mathrm{a}=4.30$ and $\mathrm{c}=7.01 \AA$. The band gaps of CdSe and $\mathrm{ZnSe}$ are $1.44 \mathrm{eV}$ and $2.18 \mathrm{eV}$, respectively, which are slightly smaller than experimental data. The calculated radiative lifetimes $(\tau)$ can be evaluated using the following equation: ${ }^{7}$

$$
\tau=2 \pi m_{e} \frac{\varepsilon \varepsilon_{0}}{e^{2}} \frac{c^{3}}{n^{3} E_{T}^{2} f}
$$

where $\varepsilon \varepsilon_{0} / e^{2}$ is the screened interaction of the exciton, $c$ is the velocity of light, $n$ is the index of refraction $(n=2.5), m_{\mathrm{e}}$ the mass of the electron, $E_{T}$ is the transition energy and $f$ is the 
transition dipole oscillator strength. The transition energy and dipole oscillator strength were calculated by the time dependent density function theory (TDDFT). ${ }^{8}$ As shown in Figure $2 \mathrm{~d}$ and Table S1, the calculated radiative lifetimes are consistent with the experimental results, which the radiative lifetimes increase with the decrease of $x$ values. With the decrease of $\mathrm{Zn}$ content ( $x$ values), their band gap decreases gradually, which causes both transition energy $E_{T}$ and transition dipole oscillator strength $f$ become smaller. According equation (4), the smaller $E_{T}$ and $f$ will lead to longer radiative lifetimes $\tau$. Hence, the PL lifetimes of $\mathrm{Zn}_{x} \mathrm{Cd}_{1-x} \mathrm{Se}$ increase with the decrease of $x$ values from 1 to 0 , as shown in Figure $2 \mathrm{~d}$ and Table S1.

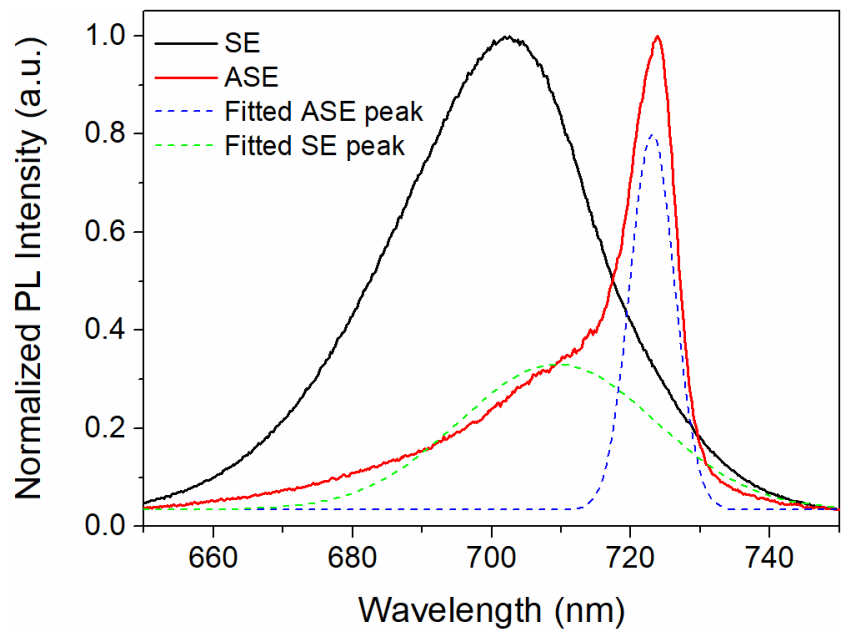

Figure S4. Spontaneous emission (SE) and amplified spontaneous emission (ASE) spectra of $\mathrm{CdSe}(x=0) \mathrm{NWs}$ in a normalized scale.
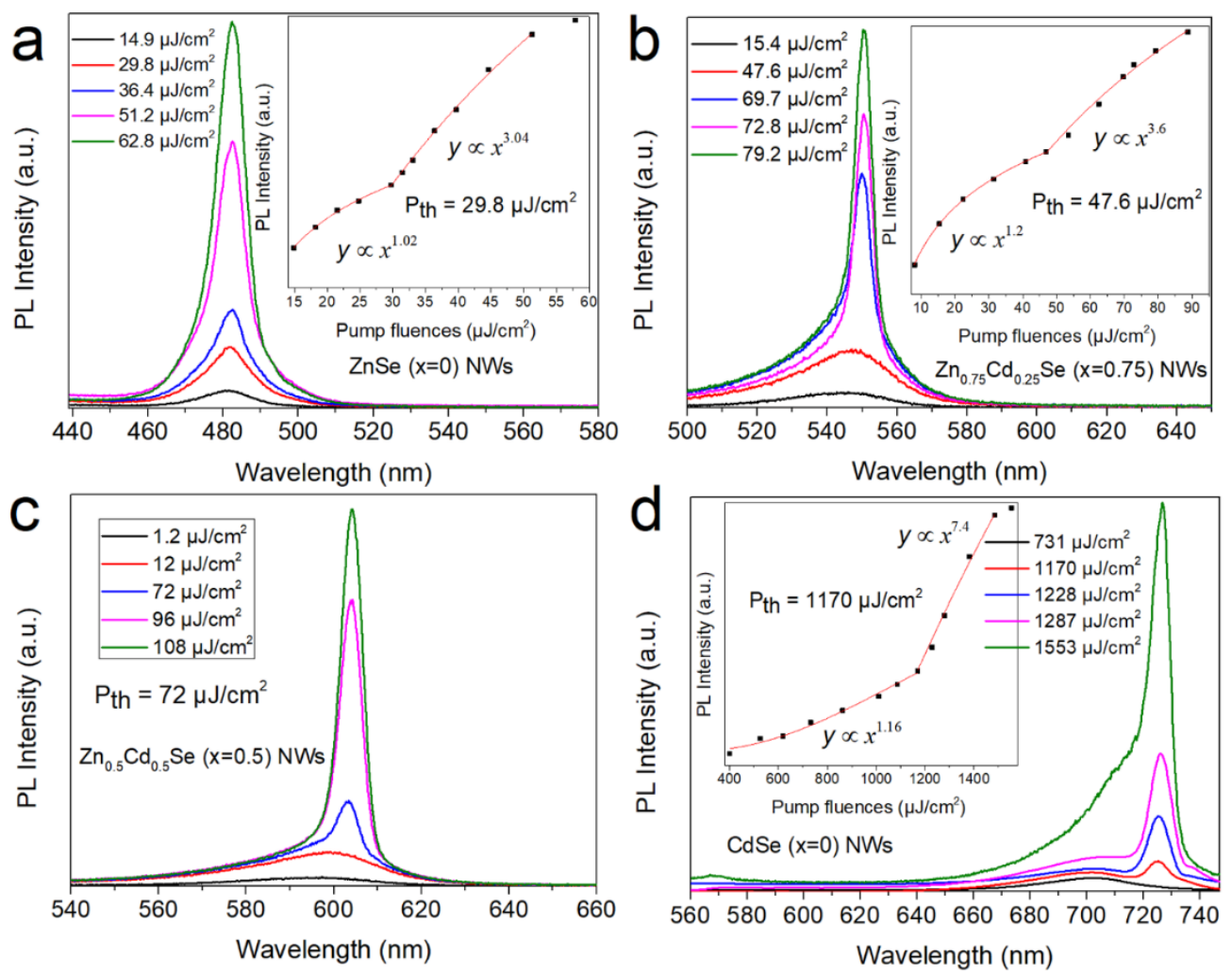
Figure S5. Power dependent spectra evolutions of $\mathrm{Zn}_{x} \mathrm{Cd}_{1-x} \mathrm{Se}$ NWs with different $x$ values under one-photon pumping of $400 \mathrm{~nm}, 1 \mathrm{kHz}$ and $150 \mathrm{fs}$ laser pluses. The insets show the power dependent PL intensity that are used to determine the ASE threshold. (a) ZnSe ( $x=1$ ) NWs; (b) $\mathrm{Zn}_{0.75} \mathrm{Cd}_{0.25} \mathrm{Se} \mathrm{NWs}$; (c) $\mathrm{Zn}_{0.5} \mathrm{Cd}_{0.5} \mathrm{Se}$ NWs; (d) CdSe ( $\left.x=0\right)$ NWs.
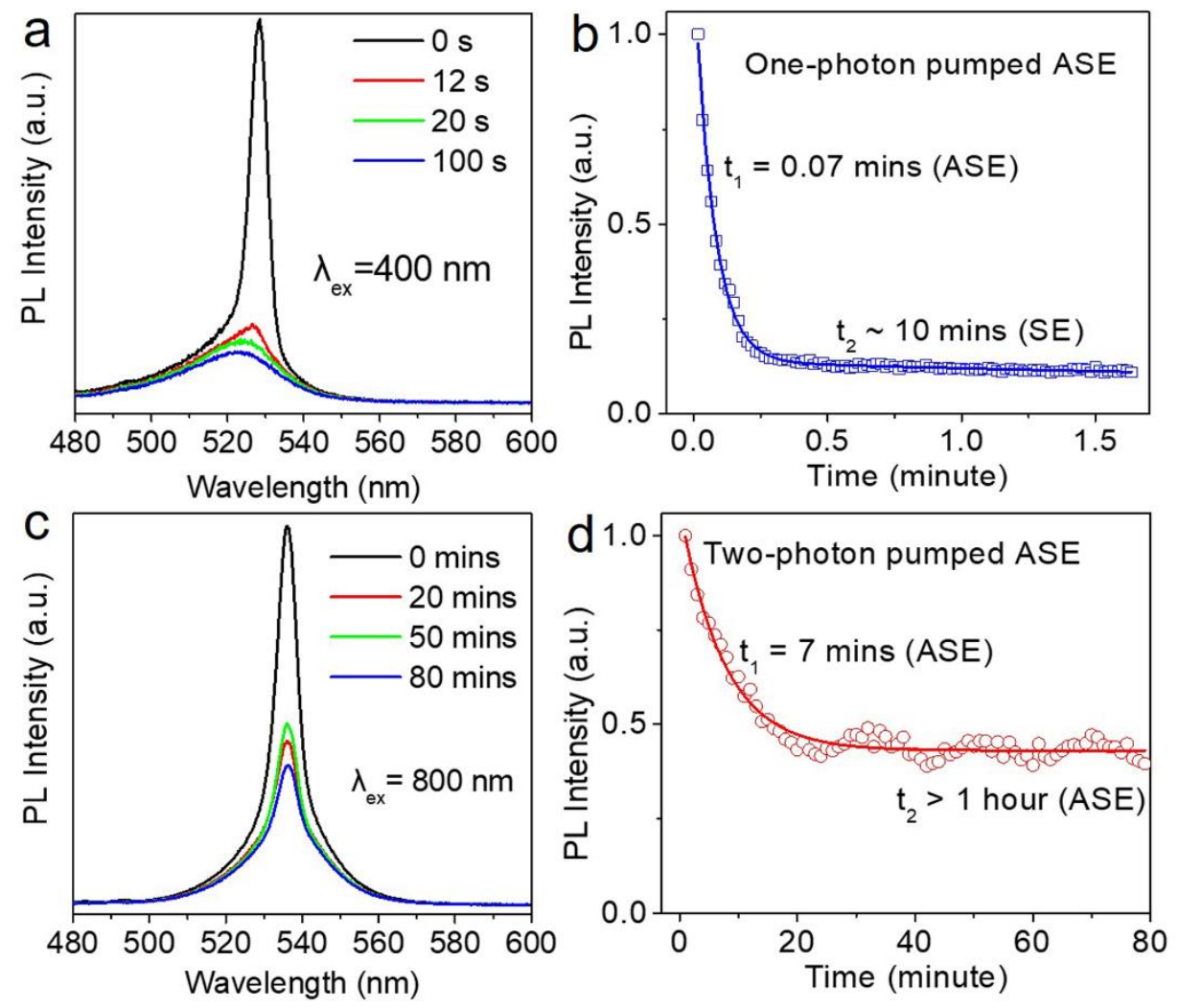

Figure S6. (a) Time dependent PL spectra of $\mathrm{Zn}_{x} \mathrm{Cd}_{1-x} \mathrm{Se}(x=0.8)$ NWs under one-photon excitation with $400 \mathrm{~nm}, 150 \mathrm{fs}$ and $1 \mathrm{kHz}$ laser pulses at a pump fluence of $50 \mu \mathrm{J} / \mathrm{cm}^{2}\left(1.25 \mathrm{P}_{\mathrm{th}}\right)$. (b) Integrated PL intensity curve depend on different pumping time upon one-photon pumped ASE from $\mathrm{Zn}_{0.8} \mathrm{Cd}_{0.2} \mathrm{Se}$. (c) PL spectra of $\mathrm{Zn}_{x} \mathrm{Cd}_{1-x} \mathrm{Se}(x=0.8) \mathrm{NWs}$ at various times after twophoto excitation with $800 \mathrm{~nm}, 150 \mathrm{fs}$ and $1 \mathrm{kHz}$ laser pulses at a pump fluence of $820 \mu \mathrm{J} / \mathrm{cm}^{2}$ (1.25 $\left.\mathrm{P}_{\text {th }}\right) .(\mathrm{d})$ Integrated PL intensity curve depend on different pumping time upon two-photon pumped ASE from $\mathrm{Zn}_{x} \mathrm{Cd}_{1-x} \mathrm{Se}$.

Figure S6a and $b$ show the change of PL intensity of one-photon pumped ASE from $\mathrm{Zn}_{x} \mathrm{Cd}_{1-}$ ${ }_{x} \mathrm{Se}$ NWs with time. The one/two-photon pumped PL intensity decay process with continuous illumination can be fitted well to a typical double-exponential decay curve since both them show two distinctly different decay tendencies. In one-photon pumped case, a very short ASE lifetime of 0.07 minutes can be observed, and then the ASE turns into spontaneous emission (SE) and continues for a short time about 10 minutes. The rapid quenching of ASE in only a few seconds indicates that the one-photon pumped ASE from $\mathrm{Zn}_{x} \mathrm{Cd}_{1-x} \mathrm{Se}$ NWs upon $400 \mathrm{~nm}$ is 
unstable. The dependence relationship between PL intensity and time of two-photon pumped ASE from $\mathrm{Zn}_{x} \mathrm{Cd}_{1-x} \mathrm{Se}$ NWs are shown in Figure S6c and d. Significantly, the two-photon pumped $\mathrm{Zn}_{x} \mathrm{Cd}_{1-x} \mathrm{Se}$ NWs exhibit stable ASE output with a long time above 1 hour. The strong intensity of ASE continually declines with 7 minutes until it keeps a weak ASE above 80 minutes with the $44 \%$ PL intensity compared with the original one. It demonstrates that the room temperature stable and tunable frequency upconverted ASE can be achieved effectively by two-photon pumping from $\mathrm{Zn}_{x} \mathrm{Cd}_{1-x} \mathrm{Se}$ NWs.

The attenuation of PL intensity for one-photon absorption (OPA) process can be expressed as follow: ${ }^{1}$

$$
\frac{d I}{d z}=-\alpha I \quad I=I_{0}-e^{\alpha z}
$$

And the attenuation of PL intensity for two-photon absorption (TPA) process can be expressed as follow: ${ }^{1}$

$$
\frac{d I}{d z}=-\beta I^{2} \quad I=\frac{I_{0}}{\left(1+I_{0} \beta z\right)}
$$

where $z$ is the penetration depth of photons (is proportional to illumination time), $I$ is the PL intensity in the depth of $z$ position of a sample; $I_{0}$ is the initial PL intensity of incident light; $\alpha$ is OPA coefficient and $\beta$ is TPA coefficient. According to the equation (5), the one-photon pumped PL intensity decay exponentially with penetration depth $z$ (illumination time), as shown in Figure S6b. Compared with equations (5) and (6), the PL intensity decay rate of two-photon pumping is significantly slower than that of one-photon pumping. Thus, the two-photon pumped ASE in $\mathrm{Zn}_{x} \mathrm{Cd}_{1-x} \mathrm{Se} \mathrm{NWs}$ is stable which can be sustained over 1 hour (Figure S6d). The carrier concentration is proportional to $I^{2}$, and the penetration depth of OPA is smaller than that of TPA because the propagating of short wavelength light is easier affected by scattering than that of long wavelength light. The surface carrier concentration of OPA is greater than that of TPA. The high active surface is more easily to react with water and oxygen that result in its instability. All these factors result in the rapid attenuation of PL intensity under one-photon pumping. Thus, it should ascribe the stable two-photon pumped ASE to the large penetration depth and low photons energy. 

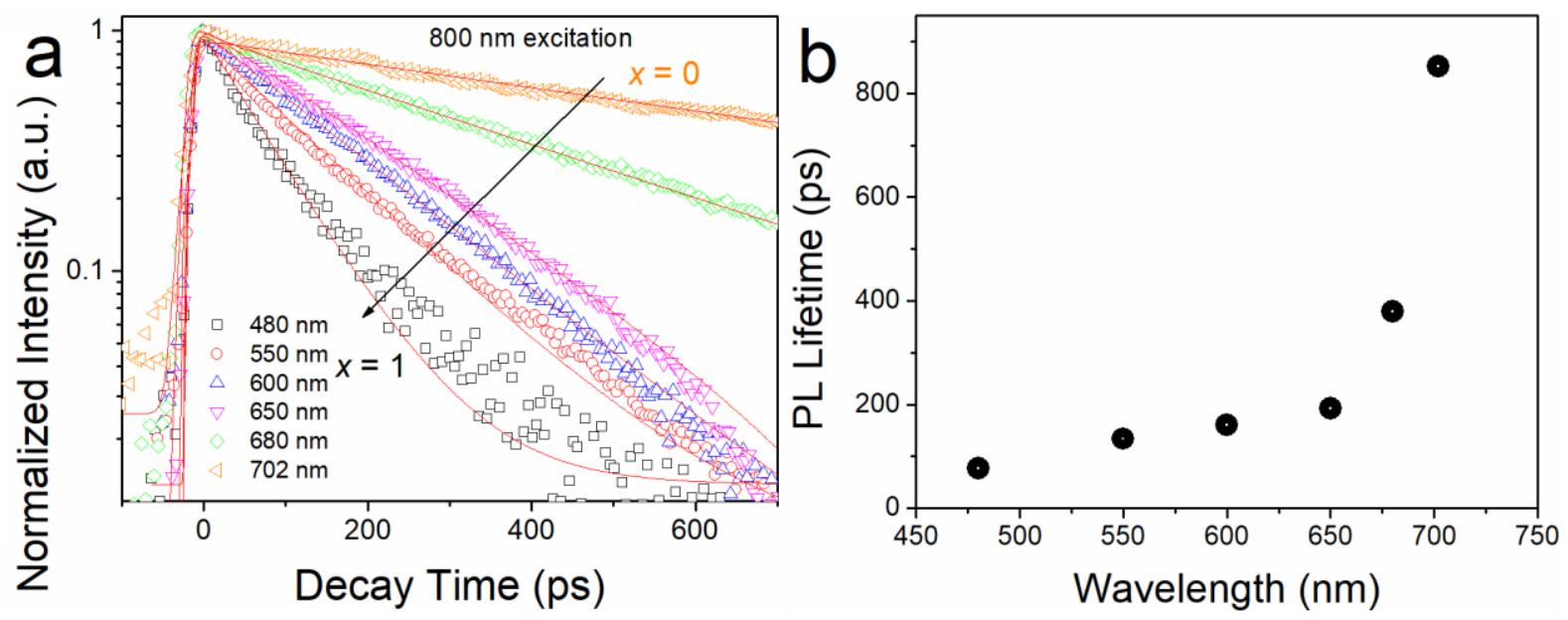

Figure S7. (a) PL decay curves of $\mathrm{Zn}_{x} \mathrm{Cd}_{1-x} \mathrm{Se}$ NWs monitored at different wavelength of 480 $\mathrm{nm}, 550 \mathrm{~nm}, 600 \mathrm{~nm}, 650 \mathrm{~nm}, 680 \mathrm{~nm}$ and $702 \mathrm{~nm}$ under pump fluence of $12 \mu \mathrm{J} / \mathrm{cm}^{2}$ of $800 \mathrm{~nm}$ excitation. (b) The corresponding fitted PL lifetime of $\mathrm{Zn}_{x} \mathrm{Cd}_{1-x} \mathrm{Se}$ NWs as a function of emission wavelength (composition).

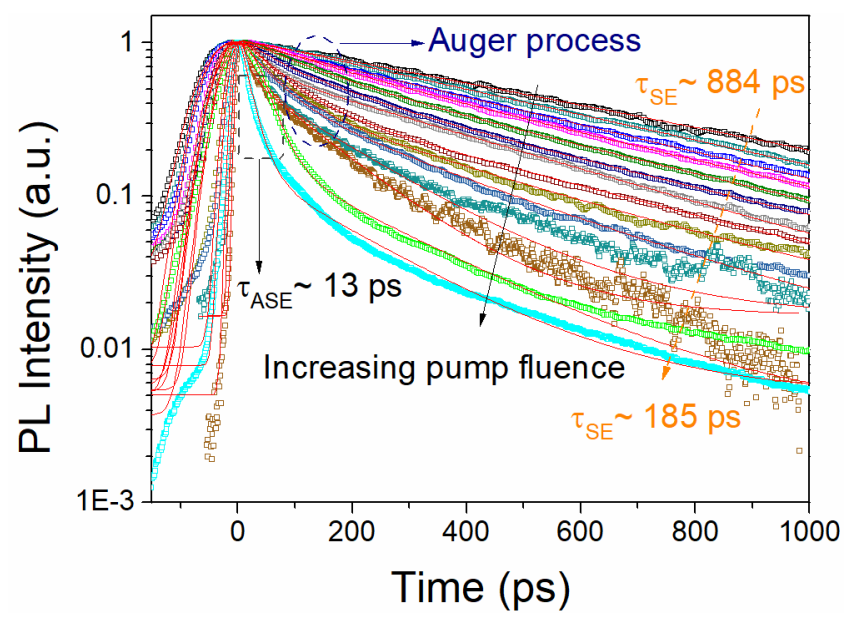

Figure S8. The pump fluence dependent TRPL spectra of $\mathrm{Zn}_{0.5} \mathrm{Cd}_{0.5} \mathrm{Se} \mathrm{NWs}$ with one-photon excitation fluence $(400 \mathrm{~nm})$.

In principle, Auger recombination process is a non-radiative recombination process that caused by multi-carrier interactions at high power pumping condition and occurred in the range from tens to hundreds of picoseconds (ps). According to the previous publications, the Auger recombination can result in the decrease of lifetime with the increase of pump power, which can be observed by time-resolved photoluminescence (TRPL) spectroscopy. ${ }^{9-12}$ Compared with TRPL method, transient absorption is a more effective and direct method to determine Auger non-radiative transition process. ${ }^{13-15}$

However, for these $\mathrm{Zn}_{x} \mathrm{Cd}_{1-x} \mathrm{Se}$ bulk alloyed NWs, it is difficult to make dense and uniform thin films for the transient absorption measurement without breaking their original morphology. In this case, we conducted power dependent TRPL measurement to discern the Auger process 
as alternative. Herein, the Auger process can be detected effectively by pump power dependent TRPL due to the appropriate instrument resolution $(<100 \mathrm{ps})$. Moreover, different types of Auger recombination mechanisms were discussed, aiming to discover the existence of excitons in NWs via fitting the pump power and time dependent carrier density curves (Figure 4a). The Auger behavior can be observed from the pump fluence dependent TRPL spectra (Figure S8). With the increase of pump fluence, the multiple charge carriers/excitons dominant Auger recombination become obvious, which results in the reduction of PL lifetime. ${ }^{9,10}$ The Auger recombination lifetimes in CdSe or ZnSe nanostructure are usually range from 45 to $360 \mathrm{ps}^{10,14-}$ ${ }^{16}$ And then, the faster exciton-exciton avalanche dominant ASE recombination emerges at the threshold pump fluence. It needs to be distinguished that the Auger recombination lifetime is significantly longer than the ASE lifetime of 13 ps (Figures $4 \mathrm{~b}$ and S8).

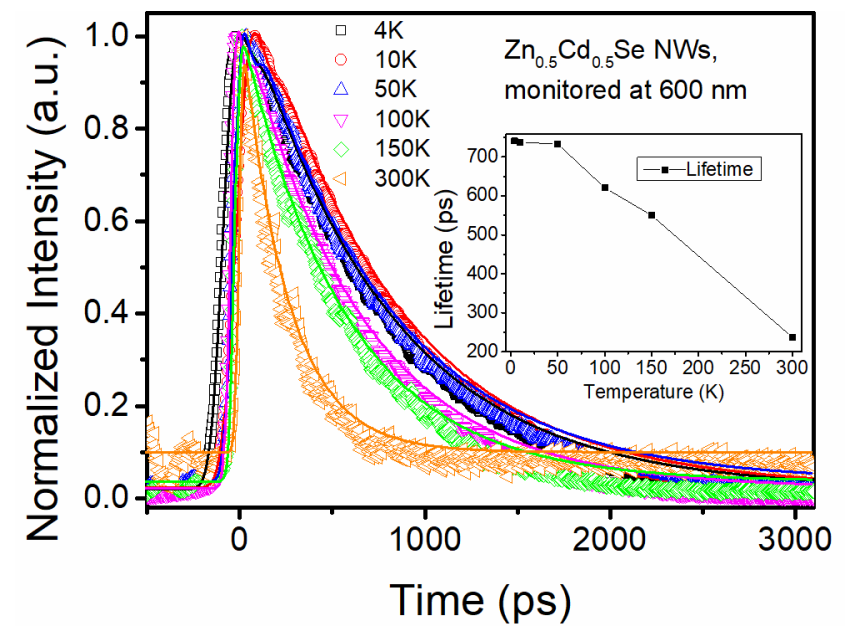

Figure S9. Temperature-dependent PL decay of $\mathrm{Zn}_{0.5} \mathrm{Cd}_{0.5} \mathrm{Se} \mathrm{NWs}$ monitored at $600 \mathrm{~nm}$, with the temperature range from $4 \mathrm{~K}$ to $300 \mathrm{~K}$. The inset shows the fitted effective lifetime of $\mathrm{Zn}_{0.5} \mathrm{Cd}_{0.5} \mathrm{Se}$ NWs with the increase of temperature.

Figure $\mathrm{S} 9$ shows the temperature-dependent carriers behaviour of $\mathrm{Zn}_{0.5} \mathrm{Cd}_{0.5} \mathrm{Se} \mathrm{NWs}$, which is evaluated by the PL decay curves. Under low temperature $(<50 \mathrm{~K})$, the effective lifetime is nearly constant with single exponential decays, which suggest that purely exciton radiative recombination dominate the decay in this regime. ${ }^{17}$ Under high temperature $(50 \mathrm{~K}-300 \mathrm{~K})$, the thermally activated nonradiative decay result in the faster decay curves.

\section{REFERENCES}

(1) Xing, G. C.; Luo, J. S.; Li, H. X.; Wu, B.; Liu, X. F.; Huan, C. H. A.; Fan, H. J.; Sum, T. C. Ultrafast Exciton Dynamics and Two-Photon Pumped Lasing from ZnSe Nanowires. Adv. Opt. Mater. 2013, 1, 319-326. 
(2) Bogani, F.; Grifoni, S.; Gurioli, M.; Morolli, L. Band-edge Dynamics and Trapping in ZnSe Crystals. Phys. Rev. B: Condens. Matter Mater. Phys. 1995, 52, 2543-2549.

(3) Gindele, F.; Woggon, U.; Langbein, W.; Hvam, J. M.; Leonardi, K.; Hommel, D.; Selke, H. Excitons, Biexcitons, and Phonons in Ultrathin CdSe/ZnSe Quantum Structures. Phys. Rev. B: Condens. Matter Mater. Phys. 1999, 60, 8773-8782.

(4) Peng, S.; Xing, G.; Tang, Z. Hot Electron-hole Plasma Dynamics and Amplified Spontaneous Emission in ZnTe Nanowires. Nanoscale, 2017, 9, 15612-15621.

(5) Perdew, J. P.; Burke, K.; Ernzerhof, M. Generalized Gradient Approximation Made Simple. Phys. Rev. Lett. 1996, 77, 3865-3868.

(6) Giannozzi, P.; Baroni, S.; Bonini ,N.; Calandra, M.; Car, R.; Cavazzoni, C.; Ceresoli, D. et. al. QUANTUM ESPRESSO: A Modular and Open-source Software Project for Quantum Simulations of Materials. J. Phys.: Condens. Matter 2009, 21, 395502.

(7) Dal Negro, L.; Yi, J. H.; Kimerling, L. C.; Hamel, S.; Williamson, A. Light Emission from Silicon-rich Nitride Nanostructures. Appl. Phys. Lett. 2006, 88, 183103.

(8) Wang, L.; Maxisch, T.; Ceder, G. O. Oxidation Energies of Transition Metal Oxides within the GGA+ U Framework. Phys. Rev. B: Condens. Matter Mater. Phys. 2006, 73, 195107.

(9) Sermage B.; Eichler H. J.; Heritage J. P.; Nelson, R. J.; Dutta, N. K. Photoexcited Carrier Lifetime and Auger Recombination in 1.3 $\mu \mathrm{m}$ InGaAsP. Appl. Phys. Lett. 1983, 42, 259-261. (10) García-Santamaría, F.; Chen, Y.; Vela, J.; Schaller, R. D.; Hollingsworth, J. A.; Klimov, V. I. Suppressed Auger Recombination in "Giant” Nanocrystals Boosts Optical Gain Performance. Nano Lett. 2009, 9, 3482-3488.

(11) Boubanga-Tombet, S.; Wright, J. B.; Lu, P.; Williams, M. R. C.; Li, C.; Wang, G. T.; Prasankumar, R. P. Ultrafast Carrier Capture and Auger Recombination in Single GaN/InGaN Multiple Quantum Well Nanowires. ACS Photonics 2016, 3, 2237-2242.

(12) Robel, I.; Bunker, B. A.; Kamat, P. V.; Kuno, M. Exciton Recombination Dynamics in CdSe Nanowires: Bimolecular to Three-Carrier Auger Kinetics. Nano Lett. 2006, 6, 1344-1349.

(13) Ben-Shahar, Y.; Philbin, J. P.; Scotognella, F.; Ganzer, L.; Cerullo, G.; Rabani, E.; Banin, U. Charge Carrier Dynamics in Photocatalytic Hybrid Semiconductor-Metal Nanorods: Crossover from Auger Recombination to Charge Transfer. Nano Lett. 2018, 18, 5211-5216. 
(14) Htoon, H.; Hollingsworth, J. A.; Dickerson, R.; Klimov, V. I. Effect of Zero- to oneDimensional Transformation on Multiparticle Auger Recombination in Semiconductor Quantum Rods. Phys. Rev. Lett. 2003, 91, 227401.

(15) Klimov, V. I.; Mikhailovsky, A. A.; McBranch, D. W.; Leatherdale, C. A.; Bawendi, M. G. Quantization of Multiparticle Auger Rates in Semiconductor Quantum Dots. Science 2000, 287, 1011-1013.

(16) Park, Y. S.; Bae, W. K.; Pietryga, J. M.; Klimov, V. I. Auger Recombination of Biexcitons and Negative and Positive Trions in Individual Quantum Dots. ACS Nano 2014, 8, 7288-7296.

(17) Donegá, C. de M.; Bode, M.; Meijerink, A. Size-and Temperature-dependence of Exciton Lifetimes in CdSe Quantum Dots. Phys. Rev. B: Condens. Matter Mater. Phys. 2006, 74, 085320 . 